\title{
Increased risk of adverse events in patients with low-on clopidogrel platelet reactivity after percutaneous coronary intervention: A systematic review and meta-analysis
}

\author{
Alexandra Bálint ${ }^{1}$, Lilla Hanák ${ }^{2}$, Péter Hegyi ${ }^{2}$, Zsolt Szakács $^{2,3}$, \\ Szimonetta Eitmann ${ }^{2}$, András Garami ${ }^{4}$, Margit Solymár ${ }^{2}$, \\ Katalin Márta ${ }^{2}, Z_{\text {Oltán Rumbus }}{ }^{4}$, András Komócsi ${ }^{1}$ \\ ${ }^{1}$ Heart Institute, Medical School, University of Pécs, Hungary \\ ${ }^{2}$ Institute for Translational Medicine, Medical School, University of Pécs, Hungary \\ ${ }^{3}$ Szentágothai Research Center, University of Pécs, Hungary \\ ${ }^{4}$ Department of Thermophysiology, Institute for Translational Medicine, \\ Medical School, University of Pecs, Hungary
}

\begin{abstract}
Background: Clinical evidence has been controversial regarding the influence of low platelet reactivity $(L P R)$, ischemic and bleeding outcomes among patients receiving coronary stent implantation. Hence, the present study performed a meta-analysis to systematically evaluate the significance of LPR on adverse cardiovascular events.
\end{abstract}

Methods: MEDLINE, EMBASE and CENTRAL databases were searched up to November 2020 for relevant studies including patients with acute coronary syndrome undergoing percutaneous coronary intervention. LPR was the exposed arm while the non-LPR group represented the control. The primary outcome of interest was bleeding risk including major and minor bleeding events. Secondary outcomes included all-cause mortality, repeated revascularization, nonfatal myocardial infarction, and stent thrombosis. Study-level outcomes were evaluated in random-effect models.

Results: A total of 20 studies with 19,064 patients were included. Pooled analysis showed that LPR was associated with an increased bleeding risk (relative risk [RR] 2.80, 95\% confidence interval [CI] $1.95-4.02, p<0.01$ ). Patients with LPR had a lower risk of non-fatal myocardial infarction (RR 0.59, $95 \%$ CI 0.38-0.91, $p<0.05$ ) and of serious vascular events (RR 0.50, 95\% CI 0.30-0.84, $p<0.01)$. Conclusions: Low platelet reactivity is associated with an increased bleeding risk of patients who underwent coronary stent implantation. The results suggest possible benefits of this marker in risk stratification, with potential improvement in risk prediction. There are potential advantages using combinations with other factors in prediction models, however, they require further study. PROSPERO registration number: CRD42019136393). (Cardiol J)

Key words: low platelet reactivity, acute coronary syndrome, percutaneous coronary intervention, bleeding risk, clopidogrel

Address for correspondence: Alexandra Bálint, MD, Heart Institute, Medical School, University of Pécs, H-7624, Pécs, Ifjúság útja 13, Hungary, tel: +36 72 536001, fax: +36 72536 387, e-mail: balint.alexandra@pte.hu

Received: 11.03.2021 Accepted: 27.06.2021 Early publication date: 2.08.2021

This article is available in open access under Creative Common Attribution-Non-Commercial-No Derivatives 4.0 International (CC BY-NC-ND 4.0) license, allowing to download articles and share them with others as long as they credit the authors and the publisher, but without permission to change them in any way or use them commercially. 


\section{Introduction}

Dual antiplatelet therapy consisting of acetylsalicylic acid and adenosine diphosphate (ADP) receptor antagonist is essential for patients undergoing percutaneous coronary intervention (PCI) [1]. Clopidogrel used to be the gold standard therapy before the introduction of new P2Y12 inhibitors, such as prasugrel and ticagrelor, which have demonstrated their clinical advantages in large randomized controlled trials (RCTs) involving acute coronary syndrome (ACS) patients $[2$, $3]$. Both prasugrel and ticagrelor provide more effective inhibition of platelet function than acetylsalicylic acid, however, their use was followed by an increased bleeding risk $[2,3]$.

Platelet function testing assesses individual response to antiplatelet drugs and platelet reactivity (PR) strongly relates to clinical outcomes after ACS [4-6]. Numerous studies have shown a relationship between high platelet reactivity (HPR) and thrombotic events [7-9]. Recent studies have also found that platelet function testing and/or genetic testing may provide important information guiding antiplatelet therapy $[10,11]$.

With the use of more effective agents, the prevalence of HPR has decreased and an increasing proportion of patients have very low on-treatment ADP reactivity. However, the clinical significance of low platelet ractivity (LPR) is less well established and it is not routinely measured. The effect of LPR was investigated in some studies raising a signal of increased bleeding risk which remains debated, partly due to contradictory results [12-14]. The objective herein, was to perform a systematic review and meta-analysis aimed at assessing the impact of LPR on efficacy and safety outcomes after PCI.

\section{Methods}

\section{Search strategy}

A systematic review and meta-analysis were performed with reference to the PRISMA guideline [15]. The National Library of Medical Publications (MEDLINE); including its subset, PubMed, the Excerpta Medica Database (EMBASE) and Cochrane Library databases were searched for relevant articles with no restriction of time in November 2020 by using a search strategy that combined the following: Medical Subject Headings and freetext search terms: "acute coronary syndrome" OR "ACS” AND "PCI" OR "percutaneous coronary intervention" AND "platelet reactivity" OR "thrombocyte reactivity". No language restriction was used. The PICO format was adapted to set the inclusion criteria. The PICO items selected were the following: $(\mathrm{P})$ patients with ACS and/or undergoing PCI and receiving dual antiplatelet therapy consisting of acetylsalicylic acid and clopidogrel, prasugrel or ticagrelor, (I) LPR (C) non-LPR or HPR based on the measurement of on-treatment PR defined by an ADP-specific platelet function assay and $(\mathrm{O})$ major adverse cardiac events (MACE) and bleeding. The non-LPR group consisted of HPR or HPR plus normal platelet reactivity (NPR) where data was given for NPR. The clinical outcomes of interest evaluated at the longest available follow-up of ADP-receptor inhibitor treatment were (a) major bleeding events (defined using the trials internal definitions using Bleeding Academic Research Consortium [BARC] 3-5 or Thrombolysis in Myocardial Infarction [TIMI] major criteria), and (b) minor bleeding events (BARC $1-2$ or TIMI minor) [16], (c) definite/probable stent thrombosis, (d) non-fatal myocardial infarction (MI) (type 1, 4a, 4b), (e) a composite endpoint of the reported serious vascular events that included cardiovascular death, non-fatal MI or non-fatal stroke, (f) repeated target vessel revascularization, and ( $\mathrm{g}$ ) all-cause mortality.

Studies that assessed responsiveness to clopidogrel, which was the difference between baseline and posttreatment PR (inhibition of platelet aggregation), were excluded from the analysis. The reference lists in the articles were also checked to capture all relevant articles published within the topic of interest.

\section{Data extraction}

Observational studies and cohorts - regardless of their prospective/retrospective design - were identified. Two investigators (A.B. and A.K.) independently screened the retrieved titles, abstracts and studies for eligibility and relevant full texts were systematically retrieved for further assessment. Disagreements between reviewers were solved by consensus. The retrieved studies were examined to exclude duplicate or overlapping data. Unpublished data and meeting abstracts were not considered for the present analysis because results could not be considered as certain and definitive.

\section{Risk of bias}

The methodological qualities of the studies were assessed using the Prediction model Risk Of Bias Assessment Toll (PROBAST) for assessing the quality of cohorts and the NewcastleOttawa Scale with reference to observational studies $[17,18]$. 


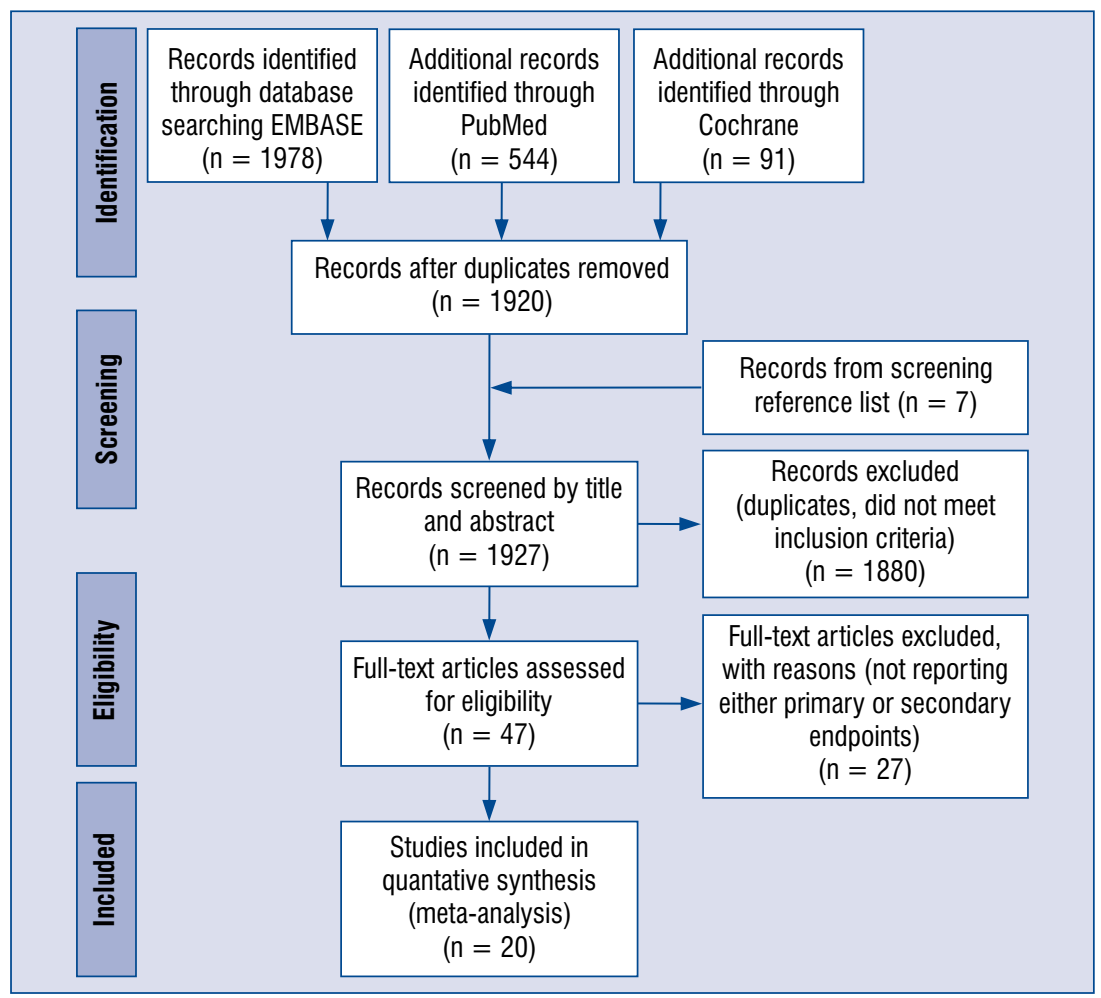

Figure 1. Preferred Reporting Items for Systematic Reviews and Meta-Analyses (PRISMA) flow diagram.

Publication bias was estimated using funnel plots. Visual evaluation and Egger's regression intercept were used to the check for asymmetry.

\section{Statistical analysis}

Statistical computations were performed using $\mathrm{R}$ ( $\mathrm{v}$ 4.0.03) package 'dmetar' designed for the evaluation of meta-analyses and OpenMeta [Analyst] open source statistical softwares. A random-effect model was applied at all the analyses with DerSimonian-Laird estimation to derive risk ratios (RR) on dichotomous outcomes and weighted mean difference on continuous data with a $95 \%$ confidence interval [CI]. Heterogeneity was tested with the $\chi^{2}$ heterogeneity statistic for which a p-value $<0.1$ was considered potentially heterogenous. Consistency was assessed using $\mathrm{I}^{2}$ statistics [19]. Sensitivity analyses were carried out omitting one study at a time and calculating the effect size with the $95 \%$ CI to investigate the influence that a single study has on the final estimation regarding LPR with increased bleeding risk.

\section{Ethical approval}

Ethical or board review approval was not required for this meta-analysis.

\section{Results}

Search results and effect of LPR on the clinical outcomes

Twenty studies, involving 19,064 patients met the inclusion criteria. The process of the literature search and bias assessment is summarized in Figure 1 and for online Supplementary Figure S4.

Table 1 describes the main characteristics of the included studies [7, 13, 20-36]. Based on pooled results of the random-effects model metaanalysis, LPR was associated with a significantly increased risk for major and minor bleeding events compared to non-LPR (RR 2.80, 95\% CI 1.95-4.02, $\mathrm{p}<0.01$ ) (Fig. 2).

Patients with LPR had significantly lower risk of non-fatal MI and of serious vascular events (RR $0.59,95 \%$ CI $0.38-0.91, \mathrm{p}<0.05$ and RR 0.50, 95\% CI 0.30-0.84, $\mathrm{p}<0.01$, respectively; Fig. 3). The risk for stent trombosis was $45 \%$ lower in the case of LPR, however, this difference did not reach the level of statistical significance (RR $0.55,95 \%$ CI $0.27-1.11, p=0.10$; Fig. 3). Even though the mortality of LPR patients was numerically higher the difference between the two groups remained insignificant (RR 1.57, 95\% CI 0.69-3.57, p = 0.28; 


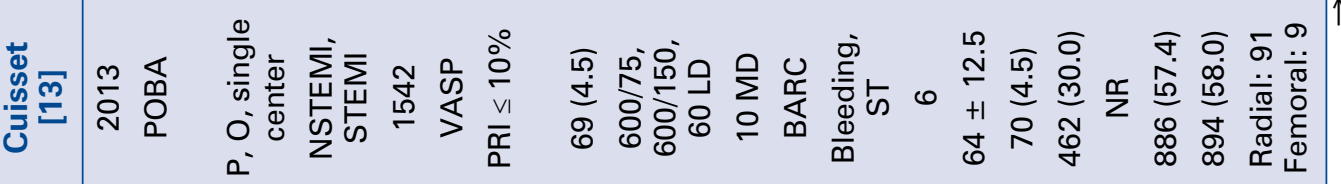

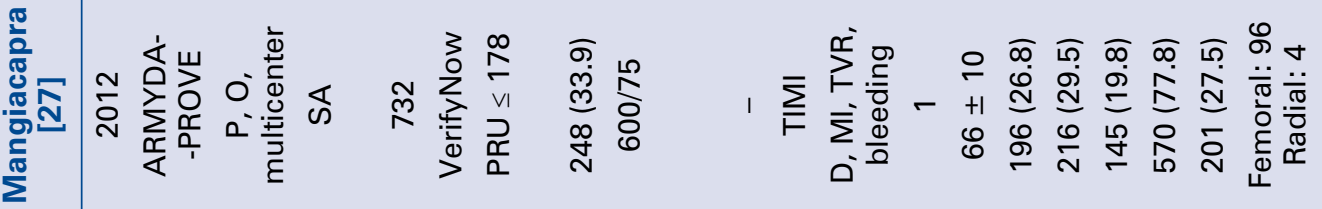

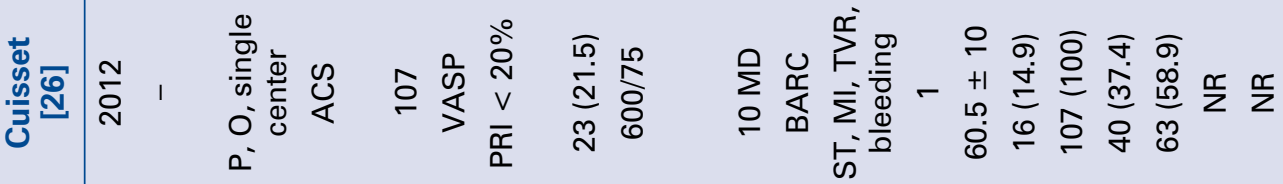

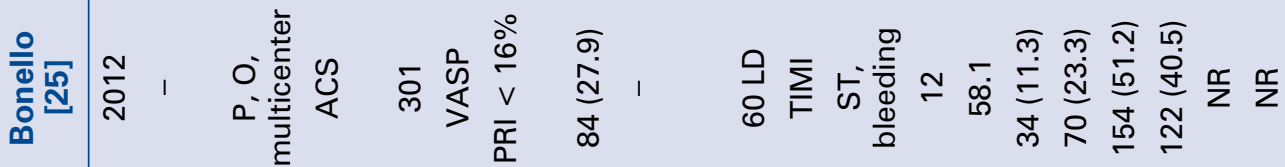

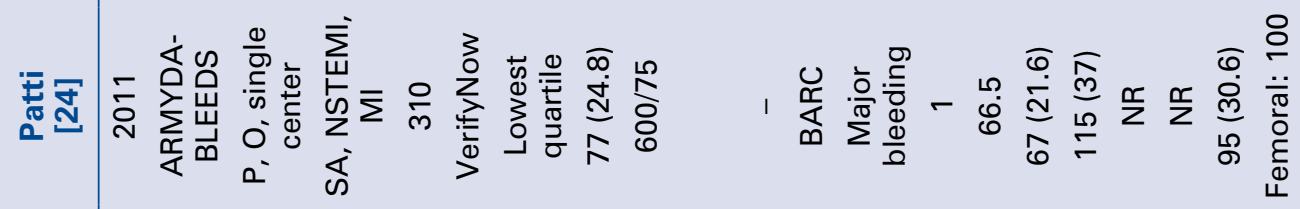

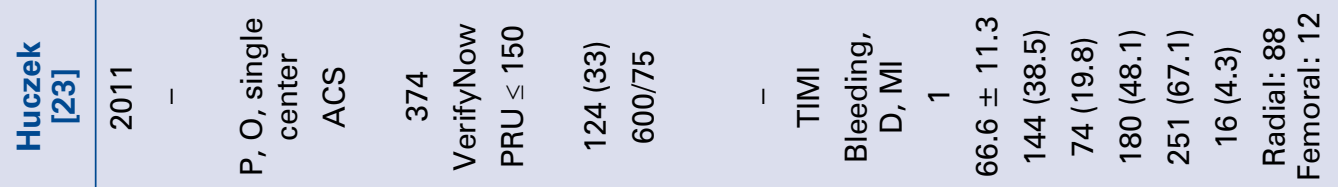

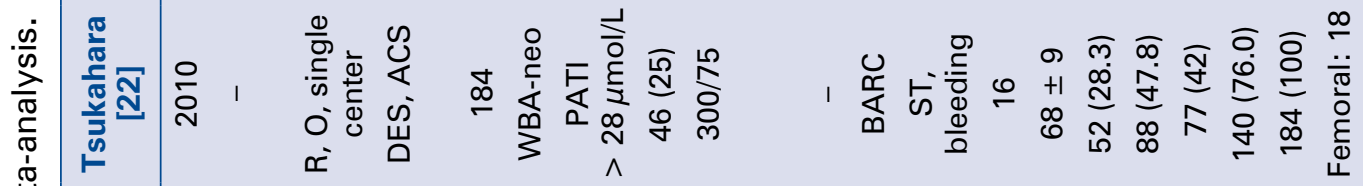

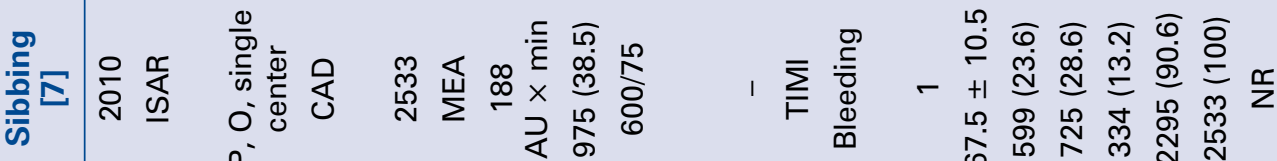

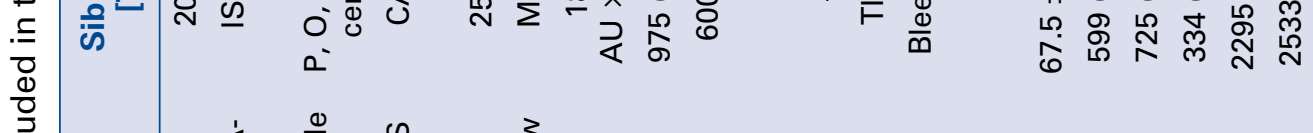

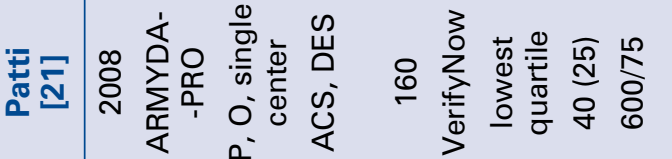

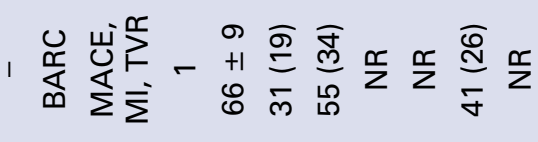

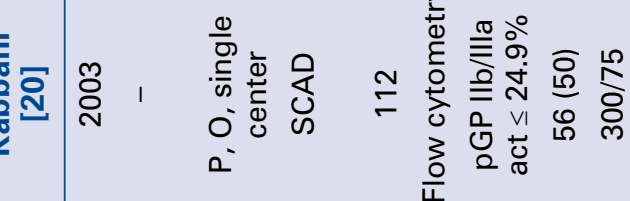
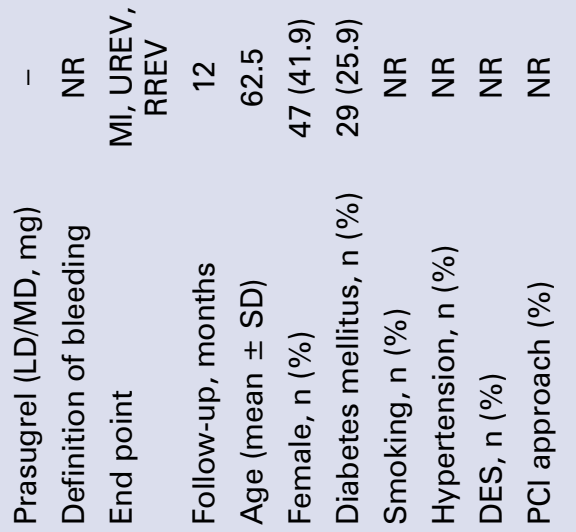


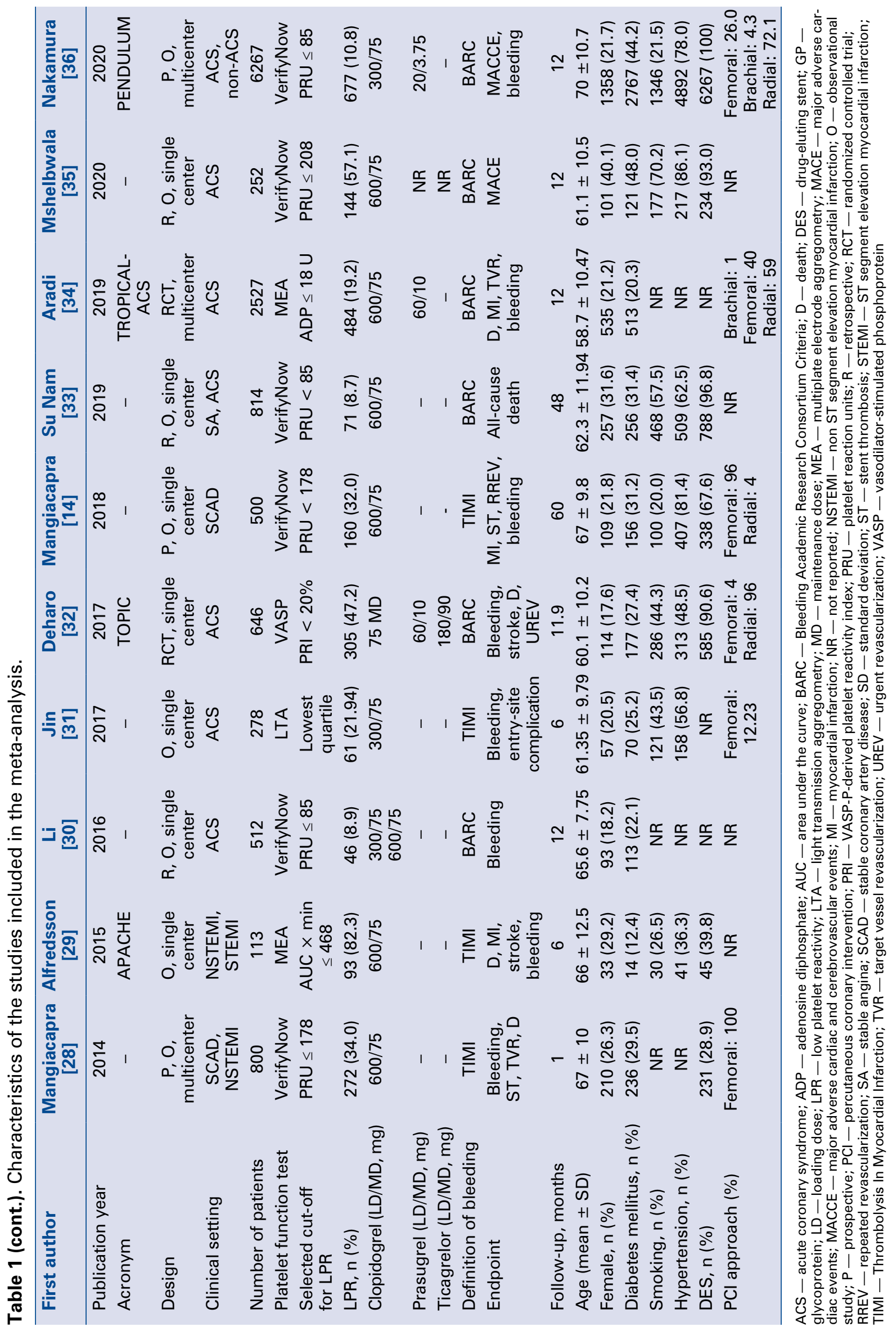




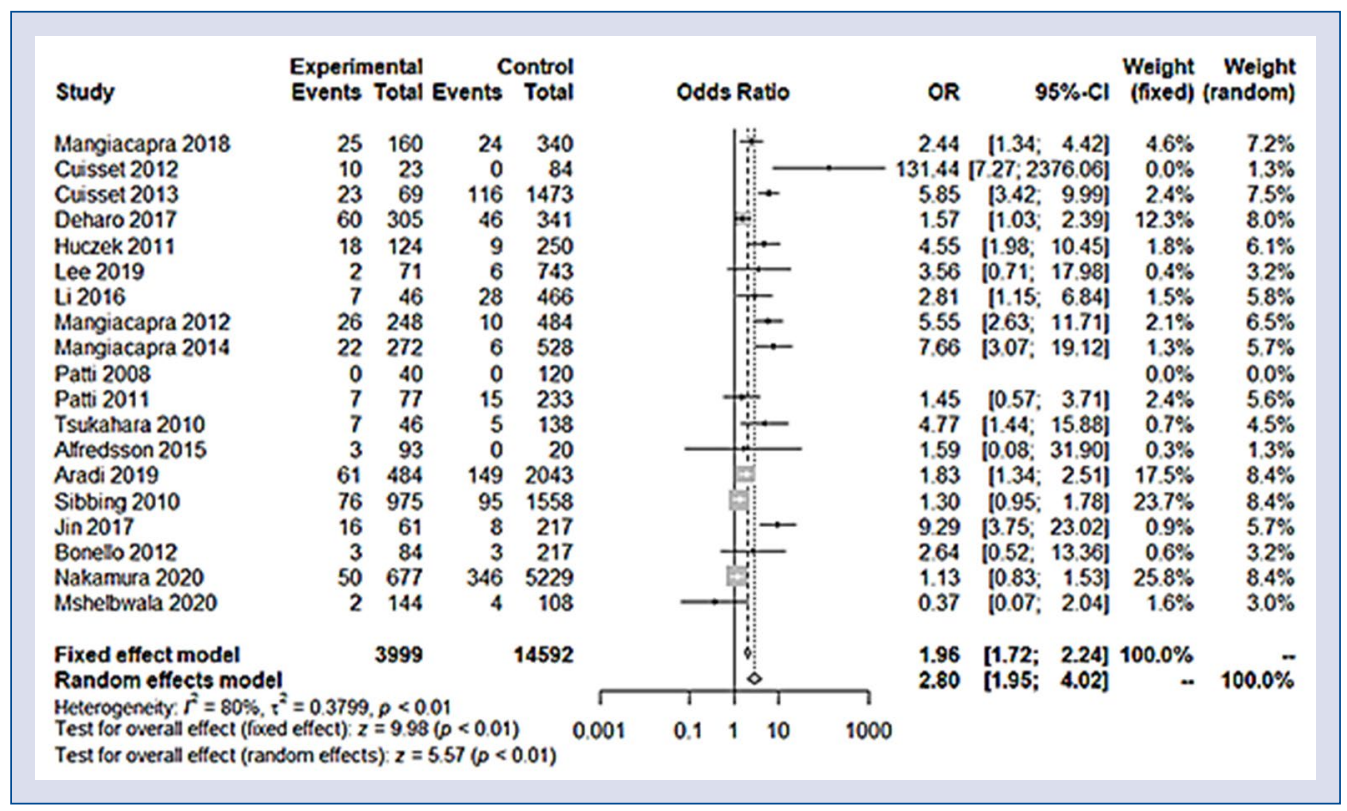

Figure 2. Principal pooled analysis. Forest plots of major and minor bleeding risk in studies following percutaneous coronary intervention with low platelet reactivity (LPR) versus without LPR. The grey rectangles are proportional with the study weight The diamond represents the cumulative odds ratio (OR) and confidence interval (Cl).

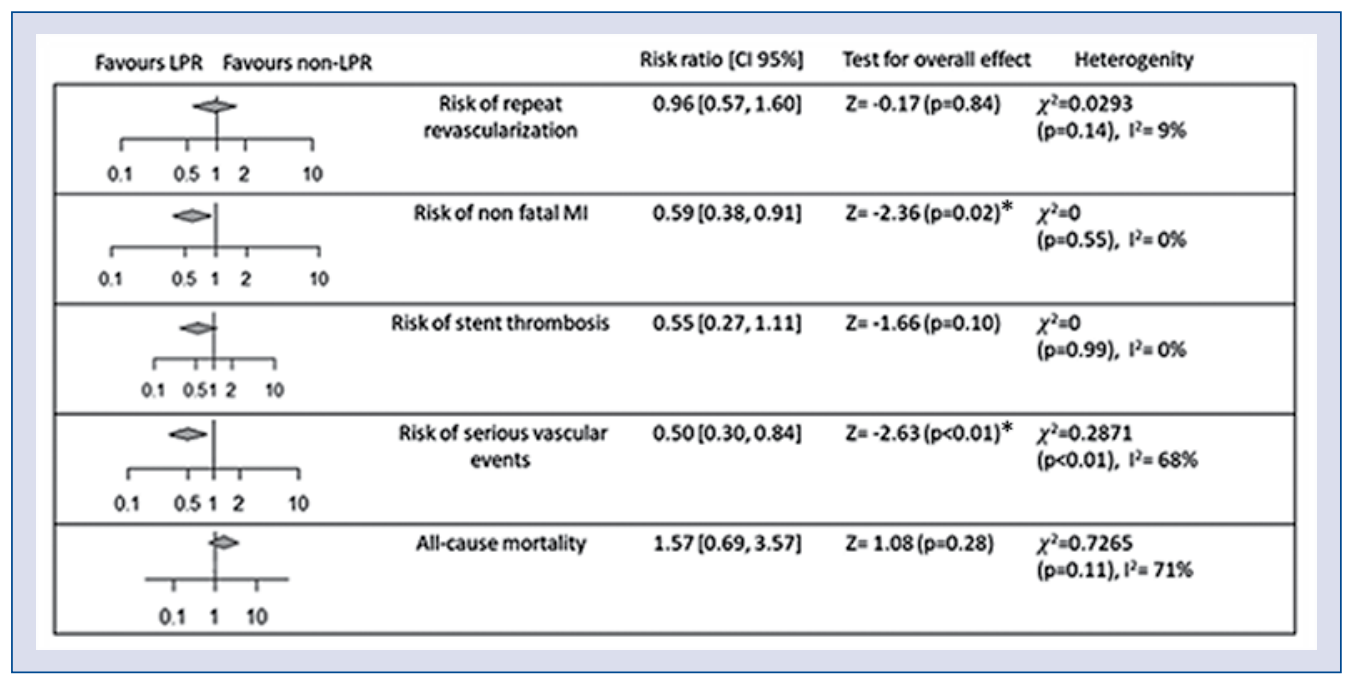

Figure 3. Summary of the outcomes of the secondary endpoints. The diamond represents the cumulative risk ratio and confidence interval $(\mathrm{Cl})$ of all patient groups. ${ }^{*}$ Mean difference $(95 \% \mathrm{Cl})$; LPR — low platelet reactivity; $\mathrm{MI}$ - myocardial infarction.

Fig. 3). No significant difference was found regarding repeated revascularization (RR $0.96,95 \% \mathrm{CI}$ $0.57-1.60, \mathrm{p}=0.84$; Fig. 3 ). Body mass index was significantly lower in the LPR group (standardized mean difference $-0.18,95 \%$ CI -0.32 to -0.05 , p $<0.01$; Suppl. Fig. S1).
Heterogeneity and subgroup analyses

The rate of LPR demonstrated a mean prevalence of $27 \%$ (95\% CI for mean $20-35 \%$, range 4.5 $-82 \%)$. Overall heterogeneity concerning major and minor bleeding events was considerable $\left(\mathrm{I}^{2}=80 \%\right.$, $\mathrm{p}<0.01)$. To find possible determinants of the 
observed heterogeneity, the prevalence of LPR and bleeding events was analyzed according to the following grouping factors: type of platelet function device, definition of bleeding events and amount of clopidogrel loading dose (LD).

The analysis confirmed that all the selected ADP-specific assays were able to predict the occurrence of bleeding events and the higher risk of patients with LPR was consistent regardless of the clinical presentation. Noticeably, considerable heterogeneity was observed in the results between studies using VASP-P and Verify Now assays; however, the Multiplate assay showed more homogenous findings (Suppl. Fig. S2). Subgroup analysis was also performed to assess the potential influence of different clopidogrel LD regimes. Despite the different types of clopidogrel loading dose, heterogeneity remained high (Suppl. Fig. S2).

When bleeding events were divided into major and minor events separately the heterogeneity was reduced considerably for major bleeding $\left(\mathrm{I}^{2}=34 \%\right)$ while heterogeneity remained high for minor bleeding $\left(I^{2}=82 \%\right.$; Suppl. Fig. S3).

\section{Publication bias}

Based on visual estimation of the funnel plot for bleeding events, no major asymmetry suggestive for publication bias was found. Furthermore, Egger's regression test confirms no small-study effect (Suppl. Fig. S4). Analysis of bias showed high quality of the source information with low probability of possible bias (Suppl. Fig. S4).

\section{Discussion}

The key finding of this meta-analysis is that patients with LPR after PCI are at a higher risk of bleeding. LPR detected by an ADP-specific laboratory assay is also associated with a lower risk of non-fatal MI. The composite endpoint of serious vascular events demonstrated lower risk with LPR. All-cause mortality did not differ significantly between LPR and non-LPR patient groups. Importantly, despite the differences in the methodology, patient selection and cut-off definition among studies, the increased risk of bleeding was homogenously reflected.

To date, this is the first meta-analysis of studies testing the role of LPR on bleeding and ischemic events in patients who underwent PCI.

In the first study reporting on the impact of enhanced response to clopidogrel treatment including 2,533 patients with coronary artery disease undergoing planned PCI, LPR was found to be associated with a two-fold higher risk for inhospital major bleeding events [7]. Further reports suggested that LPR is a marker for a higher risk of bleeding events also among prasugrel-treated patients $[25,26]$.

Some recent studies, however, do not necessarily support that optimal PR does denote the same range in every patient population. In the TRILOGY ACS trial involving ACS patients without PCI, the relationship between LPR and risks of major bleeding was missing. Among medically managed non-ST-segment elevation ACS patients receiving prolonged dual antiplatelet therapy, platelet reactivity unit values were not significantly associated with the long-term risk of major bleeding events, suggesting that LPR does not independently predict serious bleeding risk [37].

Aimed at assessing the potential influence of different clopidogrel LD regimes, a subgroup analysis was performed. The results showed no association between different LDs of clopidogrel and rate of bleeding events. These findings are in line with a recent meta-analysis that compared the use of different LDs of clopidogrel and found that these are not associated with an increased risk for major bleeding within 30 days. However, it also suggested that the administration of $600 \mathrm{mg} \mathrm{LD}$ of clopidogrel is associated with a lower risk of MACE [38]. This observation is further supported by a retrospective study of patients with stable coronary artery disease which shows no difference between different LD groups in terms of major bleeding and hemoglobin drop post PCI [39].

When interpreting data from platelet function studies, the complex mechanisms of bleeding should be considered. Besides the potential impact of platelet inhibition, several clinical factors also influence the risk of these events. Residual PR, as an independent risk factor also has several associations with patient characteristics and these may also influence the expressed risk. HPR is more frequently encountered in obese and diabetics, while LPR may more likely arise in patients with advanced age and lower body weight $[40,41]$. A significant association of LPR was revealed with lower body mass index in the current analysis. These characteristics may also impact the prognosis and when analyzed in multivariate models, the magnitude of risk, as in cases of ischemic risk with HPR, this risk is considerably reduced [42].

Importantly, periprocedural bleeding risk is substantially influenced by the access site selection, being significantly higher with transfemoral interventions. Bleeding avoidance strategies like 
routine use of the transradial approach may interfere with this risk by reducing bleeding and improving outcomes among high-risk ACS patient [43]. In the present analysis, the rate of transradial approach reached $59 \%$ (reported in 8 studies including 8,667 [45\%] patients). However, since this data was not presented in a considerable proportion of studies this impedes the further analysis of potential impact of access site selection.

The findings herein, are partly in line with the results of a previous meta-analysis published in 2015 including 17 trials with a total of 20,839 patients validating standardized cut-off points for platelet function testing. In that study thienopyridine-treated patients with HPR were associated with 2.73-fold higher risk for stent trombosis $(\mathrm{p}<0.00001)$ and a 1.5-fold higher risk for mortality $(\mathrm{p}<0.05)$ compared with those with optimal PR following PCI, meanwhile patients with LPR were associated with a 2 -fold increased risk for major bleeding complications without any further reduction in the risk of stent trombosis [38]. In the present study, there was no significant difference between LPR and non-LPR groups in case of mortality, stent trombosis or repeated revascularization. However, the risk of serious vascular events resulted in a significant difference favoring the LPR group. Regarding risk of non-fatal MI, the event rate was significantly lower in the LPR group.

However, there are some limitations that may impact the interpretation of the current results. Observational studies were included that are usually unbalanced regarding baseline clinical characteristics of the patients. These studies could reflect the real-world practice better, meanwhile due to a lack of monitoring drug compliance, underreporting negative results and incomplete follow-up, their interpretation may be more difficult and might carry ascertainment biases. To balance possible confounding factors, data were pooled with logarithmic transformation according to the random-effect model via generic inverse weighting with the intent of methodical compensation of these factors.

It should be mentioned that the patients were not treated uniformly regarding the LDs of clopidogrel and that platelet function assessments were performed at different time points after PCI with different devices and cut-offs for LPR that may have contributed to heterogeneity. There are multiple tests in the field with a real-gold standard evidently missing. Considering the plethora of available platelet function tests, the aim to restrict the analyses to those that implement a method based on $\mathrm{ADP}$ dependent in vitro platelet activation was used in order to best assess the efficacy of ADP receptor dependent activation pathway. From this perspective, acceptable methodologies were not restricted based on the final readout of the method. The use of different P2Y12 inhibitors may also have influenced residual platelet reactivity. Due to a lack of patient-level data, subgroup analyses were not done to identify drug related efficacy. It is also important to note that different definitions of bleeding may have contributed to heterogeneity. The aim to collect data according to the two most widely used and standardized definitions, the TIMI bleeding and BARC criteria were used.

\section{Conclusions}

In conclusion, this meta-analysis supports that LPR is associated with important clinical outcomes of patients who underwent coronary stent implantation. The possible benefit of this marker in risk stratification or improvement of risk prediction, if combined with other factors in prediction models remains to be established by further studies.

\section{Funding}

This study has been supported by the European Union (European Regional Development Fund) within the framework of Program Széchenyi 2020 (GINOP 2.3.2-15-2016-00048 "STAY ALIVE" and EFOP 3.6.2-16-2017-00006 “LIVE LONGER” to Péter Hegyi.

Conflict of interest: Dr. András Komócsi reports personal fees from Bayer Pharma AG, Pfizer, Krka, d. d., Merck \& Co., and Servier, outside of the submitted work. The other authors report no conflicts of interest.

\section{References}

1. Montalescot G, Sechtem U, Achenbach S, et al. Task Force Members, ESC Committee for Practice Guidelines, Document Reviewers. 2013 ESC guidelines on the management of stable coronary artery disease: the Task Force on the management of stable coronary artery disease of the European Society of Cardiology. Eur Heart J. 2013; 34(38): 2949-3003, doi: 10.1093/ eurheartj/eht296, indexed in Pubmed: 23996286.

2. Wiviott SD, Braunwald E, McCabe CH, et al. TRITON-TIMI 38 Investigators. Prasugrel versus clopidogrel in patients with acute coronary syndromes. N Engl J Med. 2007; 357(20): 2001-2015, doi: 10.1056/NEJMoa0706482, indexed in Pubmed: 17982182.

3. Wallentin L, Becker RC, Budaj A, et al. PLATO Investigators. Ticagrelor versus clopidogrel in patients with acute coronary syndromes. N Engl J Med. 2009; 361(11): 1045-1057, doi: 10.1056/NEJMoa0904327, indexed in Pubmed: 19717846. 
4. Buonamici P, Marcucci R, Migliorini A, et al. Impact of platelet reactivity after clopidogrel administration on drug-eluting stent thrombosis. J Am Coll Cardiol. 2007; 49(24): 2312-2317, doi: 10.1016/j.jacc.2007.01.094, indexed in Pubmed: 17572245.

5. Cuisset T, Cayla G, Frere C, et al. Predictive value of posttreatment platelet reactivity for occurrence of post-discharge bleeding after non-ST elevation acute coronary syndrome. Shifting from antiplatelet resistance to bleeding risk assessment? EuroIntervention. 2009; 5(3): 325-329, doi: 10.4244/51, indexed in Pubmed: 19736156.

6. Matetzky S, Shenkman B, Guetta V, et al. Clopidogrel resistance is associated with increased risk of recurrent atherothrombotic events in patients with acute myocardial infarction. Circulation. 2004; 109(25): 3171-3175, doi: 10.1161/01. CIR.0000130846.46168.03, indexed in Pubmed: 15184279.

7. Sibbing D, Schulz S, Braun S, et al. Antiplatelet effects of clopidogrel and bleeding in patients undergoing coronary stent placement. J Thromb Haemost. 2010; 8(2): 250-256, doi: 10.1111/j.1538-7836.2009.03709.x, indexed in Pubmed: 19943882.

8. Geisler T, Langer H, Wydymus M, et al. Low response to clopidogrel is associated with cardiovascular outcome after coronary stent implantation. Eur Heart J. 2006; 27(20): 2420-2425, doi: 10.1093/eurheartj/ehl275, indexed in Pubmed: 17005534.

9. Spiliopoulos S, Pastromas G, Katsanos K, et al. Platelet responsiveness to clopidogrel treatment after peripheral endovascular procedures. J Am Coll Cardiol. 2013; 61(24): 2428-2434, doi: 10.1016/j.jacc.2013.03.036.

10. Sibbing D, Aradi D, Jacobshagen C, et al. TROPICAL-ACS Investigators. Guided de-escalation of antiplatelet treatment in patients with acute coronary syndrome undergoing percutaneous coronary intervention (TROPICAL-ACS): a randomised, openlabel, multicentre trial. Lancet. 2017; 390(10104): 1747-1757, doi: 10.1016/S0140-6736(17)32155-4, indexed in Pubmed: 28855078.

11. Claassens DMF, Vos GJA, Bergmeijer TO, et al. A genotype-guided strategy for oral P2Y inhibitors in primary PCI. N Engl J Med. 2019; 381(17): 1621-1631, doi: 10.1056/NEJMoa1907096, indexed in Pubmed: 31479209.

12. Sibbing D, Schulz S, Braun S, et al. Antiplatelet effects of clopidogrel and bleeding in patients undergoing coronary stent placement. J Thromb Haemost. 2010; 8(2): 250-256, doi: 10.1111/j.1538-7836.2009.03709.x, indexed in Pubmed: 19943882.

13. Cuisset T, Grosdidier C, Loundou AD, et al. Clinical implications of very low on-treatment platelet reactivity in patients treated with thienopyridine: the POBA study (predictor of bleedings with antiplatelet drugs). JACC Cardiovasc Interv. 2013; 6(8): 854-863, doi: 10.1016/j.jcin.2013.04.009, indexed in Pubmed: 23968703.

14. Mangiacapra F, Colaiori I, Ricottini E, et al. Impact of platelet reactivity on 5-year clinical outcomes following percutaneous coronary intervention: a landmark analysis. J Thromb Thrombolysis. 2018; 45(4): 496-503, doi: 10.1007/s11239-018-1630-5, indexed in Pubmed: 29450765.

15. Hutton B, Salanti G, Caldwell DM, et al. The PRISMA extension statement for reporting of systematic reviews incorporating network meta-analyses of health care interventions: checklist and explanations. Ann Intern Med. 2015; 162(11): 777-784, doi: 10.7326/M14-2385, indexed in Pubmed: 26030634.
16. Mehran R, Rao S, Bhatt D, et al. Standardized bleeding definitions for cardiovascular clinical trials. Circulation. 2011; 123(23): 2736-2747, doi: 10.1161/circulationaha.110.009449.

17. Wolff RF, Moons KGM, Riley RD, et al. PROBAST Group. PROBAST: A Tool to Assess the Risk of Bias and Applicability of Prediction Model Studies. Ann Intern Med. 2019; 170(1): 51-58, doi: 10.7326/M18-1376, indexed in Pubmed: 30596875.

18. Wells G. A., Shea B., O'Connell D., Peterson J., Welch V., Losos M. et al. The Newcastle-Ottawa Scale (NOS) for Assessing the Quality of Nonrandomised Studies in Meta-analyses. 2000. http:// www.ohri.ca/programs/clinical_epidemiology/oxford.asp.

19. 9.5.2 Identifying and measuring heterogeneity. https://handbook-5-1.cochrane.org/chapter_9/9_5_2_identifying_and_measuring_heterogeneity.htm.

20. Kabbani SS, Watkins MW, Ashikaga T, et al. Usefulness of platelet reactivity before percutaneous coronary intervention in determining cardiac risk one year later. Am J Cardiol. 2003; 91(7): 876-878, doi: 10.1016/s0002-9149(03)00025-0, indexed in Pubmed: 12667577.

21. Patti G, Nusca A, Mangiacapra F, et al. Point-of-care measurement of clopidogrel responsiveness predicts clinical outcome in patients undergoing percutaneous coronary intervention results of the ARMYDA-PRO (Antiplatelet therapy for Reduction of MYocardial Damage during Angioplasty-Platelet Reactivity Predicts Outcome) study. J Am Coll Cardiol. 2008; 52(14): 1128-1133, doi: 10.1016/j.jacc.2008.06.038, indexed in Pubmed: 18804738.

22. Tsukahara K, Kimura K, Morita S, et al. Impact of high-responsiveness to dual antiplatelet therapy on bleeding complications in patients receiving drug-eluting stents. Circ J. 2010; 74(4): 679-685, doi: 10.1253/circj.cj-09-0601, indexed in Pubmed: 20173303.

23. Huczek Z, Filipiak KJ, Kochman J, et al. Medium on-treatment platelet reactivity to $\mathrm{ADP}$ is favorable in patients with acute coronary syndromes undergoing coronary stenting. Platelets. 2011; 22(7): 521-529, doi: 10.3109/09537104.2011.568075, indexed in Pubmed: 21443410.

24. Patti G, Pasceri V, Vizzi V, et al. Usefulness of platelet response to clopidogrel by point-of-care testing to predict bleeding outcomes in patients undergoing percutaneous coronary intervention (from the Antiplatelet Therapy for Reduction of Myocardial Damage During Angioplasty-Bleeding Study). Am J Cardiol. 2011; 107(7): 995-1000, doi: 10.1016/j.amjcard.2010.11.025, indexed in Pubmed: 21256470.

25. Bonello L, Mancini J, Pansieri M, et al. Relationship between post-treatment platelet reactivity and ischemic and bleeding events at 1-year follow-up in patients receiving prasugrel. J Thromb Haemost. 2012; 10(10): 1999-2005, doi: 10.1111/j.15387836.2012.04875.x, indexed in Pubmed: 22863374.

26. Cuisset T, Gaborit B, Dubois N, et al. Platelet reactivity in diabetic patients undergoing coronary stenting for acute coronary syndrome treated with clopidogrel loading dose followed by prasugrel maintenance therapy. Int J Cardiol. 2013; 168(1): 523-528, doi: 10.1016/j.ijcard.2012.09.214, indexed in Pubmed: 23084816.

27. Mangiacapra F, Patti G, Barbato E, et al. A therapeutic window for platelet reactivity for patients undergoing elective percutaneous coronary intervention: results of the ARMYDA-PROVE (Antiplatelet therapy for Reduction of MYocardial Damage during Angioplasty-Platelet Reactivity for Outcome Validation Effort) study. JACC Cardiovasc Interv. 2012; 5(3): 281-289, doi: 10.1016/j.jcin.2012.01.009, indexed in Pubmed: 22440493.

28. Mangiacapra F, Cavallari I, Barbato E, et al. Impact of chronic kidney disease on platelet reactivity and outcomes of patients re- 


\section{Cardiology Journal}

ceiving clopidogrel and undergoing percutaneous coronary intervention. Am J Cardiol. 2014; 113(7): 1124-1129, doi: 10.1016/j. amjcard.2013.12.018, indexed in Pubmed: 24507863.

29. Alfredsson J, Lindahl TL, Gustafsson KM, et al. Large early variation of residual platelet reactivity in Acute Coronary Syndrome patients treated with clopidogrel: results from Assessing Platelet Activity in Coronary Heart Disease (APACHE). Thromb Res. 2015; 136(2): 335-340, doi: 10.1016/j.thromres.2015.05.021, indexed in Pubmed: 26033398.

30. Li S, Liu H, Liu J. Predictive performance of adding platelet reactivity on top of CRUSADE score for 1-year bleeding risk in patients with acute coronary syndrome. J Thromb Thrombolysis. 2016; 42(3): 360-368, doi: 10.1007/s11239-016-1366-z, indexed in Pubmed: 27113341.

31. Jin L, Yu H, Dong T, et al. The prognostic value of ADP-induced platelet aggregation for bleeding complications in low: intermediate risk patients with acute coronary syndrome taking clopidogrel after percutaneous coronary intervention. Heart Lung Circ. 2017; 26(1): 49-57, doi: 10.1016/j.hlc.2016.05.113, indexed in Pubmed: 27451349.

32. Deharo P, Quilici J, Camoin-Jau L, et al. Benefit of switching dual antiplatelet therapy after acute coronary syndrome according to on-treatment platelet reactivity: the TOPIC-VASP pre-specified analysis of the topic randomized study. JACC Cardiovasc Interv. 2017; 10(24): 2560-2570, doi: 10.1016/j.jcin.2017.08.044, indexed in Pubmed: 29268886.

33. Su Nam L, Moon D, Sung MK, et al. Impact of platelet reactivity on long-term prognosis in Korean patients receiving percutaneous coronary intervention. Platelets. 2019; 30(8): 1030-1035, doi: 10.1080/09537104.2018.1562172, indexed in Pubmed: 30601072.

34. Aradi D, Gross L, Trenk D, et al. Platelet reactivity and clinical outcomes in acute coronary syndrome patients treated with prasugrel and clopidogrel: a pre-specified exploratory analysis from the TROPICAL-ACS trial. Eur Heart J. 2019; 40(24): 1942-1951, doi: 10.1093/eurheartj/ehz202, indexed in Pubmed: 31226213.

35. Mshelbwala FS, Hugenberg DW, Kreutz RP. Intensified P2Y12 inhibition for high-on treatment platelet reactivity. J Thromb Thrombolysis. 2020; 50(3): 619-627, doi: 10.1007/s11239-02002075-x, indexed in Pubmed: 32152791.

36. Nakamura M, Kadota K, Takahashi A, et al. PENDULUM Registry Investigators*. Relationship Between Platelet Reactivity and Ischemic and Bleeding Events After Percutaneous Coronary Intervention in East Asian Patients: 1-Year Results of the PENDULUM Registry. J Am Heart Assoc. 2020; 9(10): e015439, doi: 10.1161/JAHA.119.015439, indexed in Pubmed: 32394794.

37. Cornel JH, Ohman EM, Neely B, et al. Relationship of platelet reactivity with bleeding outcomes during long-term treatment with dual antiplatelet therapy for medically managed patients with non-ST-segment elevation acute coronary syndromes. J Am Heart Assoc. 2016; 5(11), doi: 10.1161/JAHA.116.003977, indexed in Pubmed: 27815268.

38. Aradi D, Kirtane A, Bonello L, et al. Bleeding and stent thrombosis on P2Y12-inhibitors: collaborative analysis on the role of platelet reactivity for risk stratification after percutaneous coronary intervention. Eur Heart J. 2015; 36(27): 1762-1771, doi: 10.1093/ eurheartj/ehv104, indexed in Pubmed: 25896078.

39. Piqueras-Flores J, Jurado-Román A, López-Lluva MT, et al. Efficacy and safety of loading doses with P2Y12-receptor antagonists in patients without dual antiplatelet therapy undergoing elective coronary intervention. J Cardiovasc Pharmacol. 2019; 73(1): 56-59, doi: 10.1097/FJC.0000000000000632, indexed in Pubmed: 30383607.

40. Tekkeşin Aİ, Kaya A, Çakıllı Y, et al. The first six-month clinical outcomes and risk factors associated with high on-treatment platelet reactivity of clopidogrel in patients undergoing coronary interventions. Anatol J Cardiol. 2016; 16(12): 967-973, doi: 10.14744/AnatolJCardiol.2016.6855, indexed in Pubmed: 27271476.

41. Wakabayashi S, Ariyoshi N, Kitahara H, et al. Efficacy of 2.5-mg prasugrel in elderly or low-body-weight patients. Circ J. 2018; 82(9): 2326-2331, doi: 10.1253/circj.CJ-18-0337, indexed in Pubmed: 29962391.

42. Droppa M, Tschernow D, Müller KAL, et al. Evaluation of clinical risk factors to predict high on-treatment platelet reactivity and outcome in patients with stable coronary artery disease (PREDICT-STABLE). PLoS One. 2015; 10(3): e0121620, doi: 10.1371/journal.pone.0121620, indexed in Pubmed: 25799149.

43. Komócsi A, Aradi D, Kehl D, et al. Meta-analysis of randomized trials on access site selection for percutaneous coronary intervention in ST-segment elevation myocardial infarction. Arch Med Sci. 2014; 10(2): 203-212, doi: 10.5114/aoms.2014.42570, indexed in Pubmed: 24904651. 